\title{
ANÁLISE MULTIVARIADA DA DIVERGÊNCIA GENÉTICA EM 29 POPULAÇÕES DE CUBIU (Solanum sessiliflorum Dunal) AVALIADA NA ZONA DA MATA DO ESTADO DE PERNAMBUCO.*
}

\author{
Danilo Fernandes da SILVA FILHO', Clodoaldo José da ANUNCIAÇÃO \\ FILHO ${ }^{2}$, Hiroshi NODA ${ }^{1}$, Odemar Vicente dos REIS ${ }^{3}$
}

\begin{abstract}
RESUMO - A amplitude da variabilidade genética em 29 populações de cubiu do Programa de Melhoramento Genético de Hortaliças do Instituto Nacional de Pesquisas da Amazônia-INPA, foi avaliada num experimento conduzido na Estação Experimental da Empresa Pernambucana de Pesquisa Agropecuária-IPA, em Vitória de Santo Antão, Estado de Pernambuco. Adotou-se o delineamento experimental em blocos casualizados com 4 repetições. Coletaram-se dados referentes a: largura do fruto $(\mathrm{cm})$; comprimento do fruto $(\mathrm{cm})$; diâmetro do colo $(\mathrm{cm})$; área da folha $\left(\mathrm{cm}^{2}\right)$; altura da planta $(\mathrm{cm})$; número total de frutos/planta; peso médio de frutos/planta $(\mathrm{g})$; produção estimada de frutos (ton/ha); número de lóculos; espessura da polpa $(\mathrm{mm})$ e de solîdos solúveis totais (\%). A análise de agrupamento, pelo Método de Otimização de Tocher, usando a Distância Generalizada de Mahalanobis, agrupou as 29 populações de cubiu em 9 diferentes grupos. Entre os pares de menor e maior divergências genéticas, foram identificadas as populações procedentes de Umariaçu (AM) e de São Paulo de Olivença (AM) e as de Borba (AM) e de São Gabriel da Cachoeira (AM), respectivamente. As populações originárias de Ataláia do Norte (AM), Borba (AM), Iquitos (Peru), São Gabriel da Cachoeira (AM) e de Belém (PA) apresentaram as maiores distâncias genéticas entre os grupos formados. Portanto, podem ser indicadas como progenitores potenciais em programa de melhoramento genético do cubiu.
\end{abstract}

Palavras chave: Cubiu, Solanum sessiliflorum, Solanaceae, divergência genética, melhoramento Multivariate Anaysis of the Genetic Divergence in 29 Populations of Cubiu (Solanum sessiliflorum) Evaluated in the Zona da Mata of the State of Pernambuco.

ABSTRACT - The amplitude of the genetic divergence among 29 populations of cubiu from the germplasm collection maintaned by the Amazonian National Research Institute (INPA) was evaluated. The trial was carried out in the Vitória de Santo Antāo Experiment Station of the Pernambuco State Research Enterprise (IPA), Pernambuco, Brazil. The experimental design was a randomized complete block, with four replications. The following characters of agronomic importance were evaluated: fruit width $(\mathrm{cm})$; fruit length $(\mathrm{cm})$; stem diameter at soil level $(\mathrm{cm})$; leaf area $\left(\mathrm{cm}^{2}\right)$; plant height $(\mathrm{cm})$; total fruit number/plant; average fruit weight/ plant $(\mathrm{g})$; estimated fruit yield (Mt/ha); loculi number; pulp thickness ( $\mathrm{mm}$ ) and total soluble solids (\%). Cluster analysis (Tocher Optimization Method, using the Mahalanobis Generalized Distance) grouped the 29 cubiu accessions into 9 groups. The accessions from Umariaçú (AM) and São Paulo de Olivença (AM) were most closely related, and are also geographically close, and those from Borba (AM) and São Gabriel da Cachoeira (AM) were most divergent, both genetically and geographically. The accessions from Atalaia do Norte (AM), Borba (AM), Iquitos (Peru), São Gabriel da Cachoeira (AM) and Belém (PA) presented good agronomic traits and were divergent among themselves. Their use as potential progenitors in the cubiu breeding program may provide both good quality and heterosis.

Key-words: Orinoco apple, Solanum sessiliflorum, Solanaceae, genetic divergence, breeding

INPA / CPCA - C.P. 478, 69.011-970 - Manaus, Amazonas, Brasil.

2 Universidade Federal Rural de Pernambuco, Departamento de Fitotecnia, 51.171-030, Recife, Pernambuco, Brasil.

3 Empresa Pernambucana de Pesquisa Agropecuária, C.P. 1022, 50.000-000, Recife, Pernambuco, Brasil. Parte da dissertação de mestrado do primeiro autor. 


\section{INTRODUÇÃO}

O cubiu (Solanum sessiliflorum), é uma hortaliça nativa da Amazônia que produz frutos comestíveis com um conteúdo considerável de nutrientes. No século passado, antes da introdução do limão e do tomate na Amazônia, o cubiu era muito utilizado pela população local. Atualmente, é cultivado em pequena escala pelos agricultores tradicionais do Amazonas. Como alimento, é consumido in natura, e nas formas de sucos, doces e geléia. Na culinária, como tempero de pratos à base de peixe, carne e frango. Sua principal utilização na medicina tradicional, tem sido para controlar altos níveis de colesterol, ácido úrico e açúcar no sangue.

A partir do ano de 1975, os pesquisadores do Instituto Nacional de Pesquisas da Amazônia-INPA, constituiram uma coleção de cubiu da qual caracterizaram e avaliaram vários acessos por meio de técnicas de análise estatística univariada (PAHLEN 1977; SILVA FILHO et al., $1989 ; 1990 ; 1993)$. A análise multivariada tem sido uma técnica eficiente para o estudo de divergências genéticas. No momento atual, o emprego dessa técnica foi facilitado com o surgimento de programas específicos para uso em microcomputadores. Com esse recurso os melhoristas podem facilmente classificar os genótipos em grupos e escolher progenitores que serão envolvidos em cruzamentos, visando a obtenção de cultivares com características superiores àquelas já existentes. Dentre as várias técnicas utilizadas pelos melhoristas, o Método de Otimização de Tocher, baseado na Distância Generalizada de Mahalanobis, é o mais recomendado (BAHTT, 1970; BEHL et al., 1985; OLIVEIRA, 1989; SHAMSUNDDIN, 1985; SINGH, 1984).

Este trabalho tem o obejetivo de estimar a amplitude da divergência genética em populações cultivadas de cubiu da Amazônia.

\section{MATERIAL E MÉTODOS}

O germoplasma utilizado no experimento, consta de 29 populações de cubiu, originárias de diversas partes da Amazônia brasileira, peruana e colombiana (Fig.1).

$\mathrm{O}$ experimento foi conduzido na Estação Experimental da Empresa Pernambucana de Pesquisa AgropecuáriaIPA em Vitória de Santo Antão, Estado de Pernambuco.

O clima local é classificado como $\mathrm{C}_{1} \mathrm{~S}_{2} \mathrm{~A} \cdot \mathrm{a}$ seco sub-úmido megatérmico no esquema de Thonrthwaith. A precipitação apresenta valores médios máximos entre os meses de março e julho (70\% do total anual). O equilíbrio hídrico é apenas atingido (quando não há excesso nem deficiência) entre os meses de abril e julho. Do mês de setembro até março vão se acumulando deficiências hídricas, que apresentam valores críticos nos meses de novembro e dezembro (ENCARNAÇÃO, 1980).

O solo predominante na região é formado por uma associação de: Latossolo Vermelho-Amarelo Distrófico com A moderado, textura argilosa; Podzólico Vermelho-Amarelo Orto, relevo forte ondulado; Podzólico 


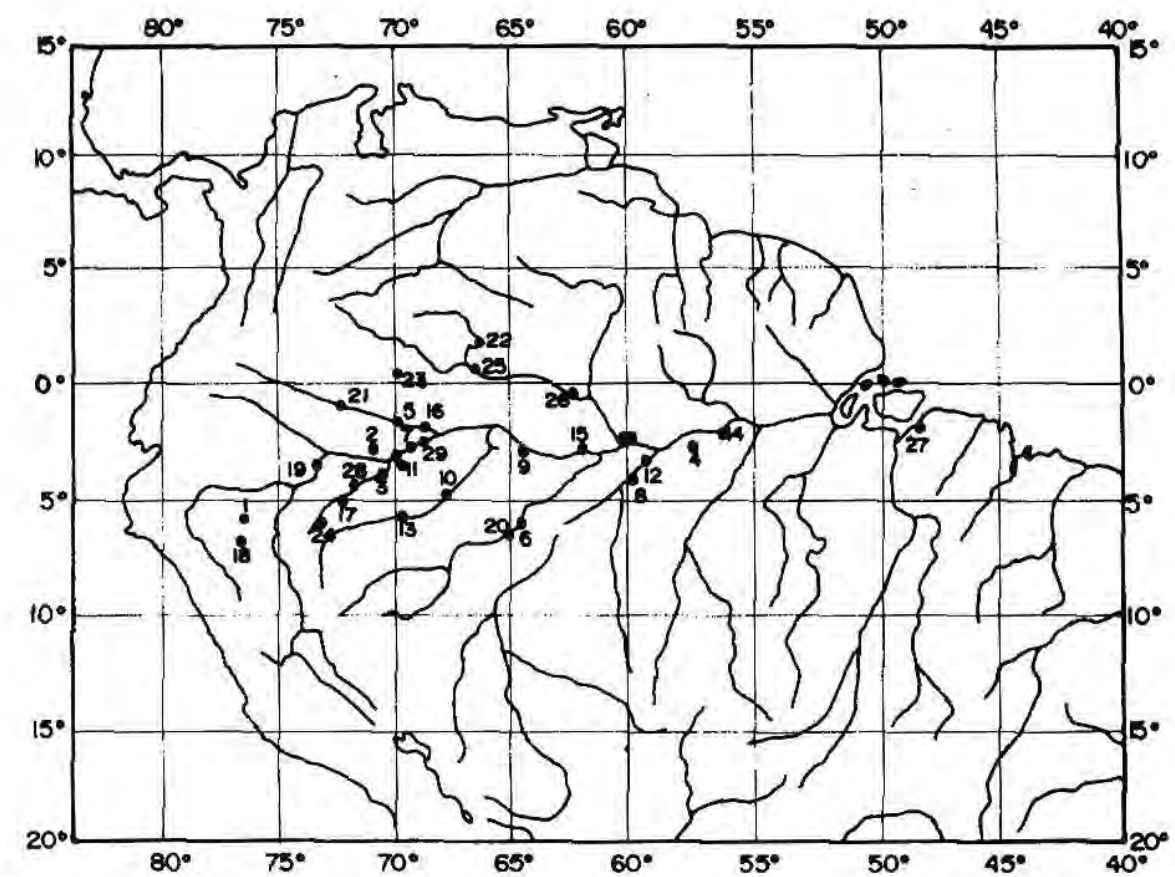

Figura 1. Origem geográfica de 29 populações de cubiu ( $S$. sessiliflorum) avaliadas na Zona da Mata do Estado de Pernambuco em 1992/1993. 1.Yurimaguas; 2. Arara; 3. Ataláia do Norte; 4. Ponta Alegre; 5. Ipiranga; 6. Canutama; 7. Umariaçú; 8. Borba; 9. Tefé; 10. Carauari; 11. Benjamin Constant; 12. Nova Olinda; 13. Eirunepé; 14. Parintins; 15. Coari; 16. Betânia; 17. Estirão do Equador; 18. Tarapoto; 19. Iquitos; 20. Lábrea; 21. Putumayo; 22. Cucui; 23. Vila Bitencourt; 24. Palmeiras; 25. São Gabriel da Cachoeira; 26. Barcelos; 27. Belém; 28. Mayuruna; e 29. São Paulo de Olivença.

Vermelho-Amarelo Latossólico, textura argilosa, relevo forte ondulado e montanhoso (CONDEPE, 1987).

A semeadura foi feita no dia 11.07.92. Utilizou-se bandejas de isopor com solo previamente autoclavado a $120^{\circ} \mathrm{C}$, por duas horas. As bandejas foram colocadas em casa de vegetação.

No $30^{\circ}$ dia após a semeadura, foi realizada a repicagem das plantas para bandejas com tubetes mais espaçosas. Colocou-se somente uma planta por tubete.

O preparo da área experimental foi realizado no fim do mês de agosto de 1992, constando de uma aração e uma gradagem.

A adubação fundamental na cova consistiu de $70 \mathrm{~g}$ de superfosfato simples, $50 \mathrm{~g}$ de cloreto de potássio e $20 \mathrm{~g}$ de sulfato de amônio.

O plantio definitivo foi realizado no dia 11.09.92. Aos quinze dias após o transplante, foi feita uma adubação em cobertura com $10 \mathrm{~g}$ de úreia/planta, repetindo-se mensalmente até o dia 11.01.93.

Em virtude da ocorrência de ácaros, pulgões e vaquinhas atacando as plantas, houve a necessidade do controle dessas pragas. Os ácaros foram controlados com pulverizações de Dicofol (Kelthane $185 \mathrm{E}$ ) na proporção de $20 \mathrm{ml} / 20$ litros d'água. Os pulgões, com a aplicação de Methamidophos 
(Tamaron $600 \mathrm{E}$ ) e as Vaquinhas com a utilização de Azimphos Ethil (Gusathion 400).

Adotou-se o delineamento experimental em blocos casualizados com 29 tratamentos (populações) em quatro repetições. A unidade experimental constituiu-se de 5 plantas úteis distribuídas numa área de $7,5 \mathrm{~m}^{2}$ obedecendo o espaçamento de $1,00 \mathrm{~m}$ entre as plantas e $1,50 \mathrm{~m}$ entre as fileiras.

As avaliações foram realizadas no campo e no laboratório. Em campo, efetuou-se as mensurações dos caracteres da parte vegetativa das plantas. No laboratório, foram avaliadas as características dos frutos.

Procederam-se as avaliações com base em cinco plantas úteis de cada parcela. Os dados foram anotados individualmente por planta e depois calculadas as médias aritméticas.

Os seguintes caracteres foram avaliados:

a) O diâmetro do colo= $\mathrm{DC}(\mathrm{cm})$, medido com um paquímetro na secção transversal do colo das plantas, a uma distância de $3 \mathrm{~cm}$ da superfície do solo. Essa avaliação foi realizada aos 120 dias após o transplante.

b) A altura da planta=AP $(\mathrm{cm})$, medida com um metro de madeira na parte compreendida entre o nível da superfície do solo e a gema apical da planta. A avaliação foi efetuada aos 120 dias após o transplante.

c) A área da folha=AF $\left(\mathrm{cm}^{2}\right)$, medida com uma régua graduada, $\mathrm{e}$ obtida ao multiplicar-se o valor da largura máxima pelo comprimento máximo de uma folha considerada adulta, localizada no quinto internódio do caule de cada planta. Posteriormente foram utilizadas equações alométricas para estimar a área mais aproximada do real, de acordo com a metodologia de EVANS (1972). Essa avaliação foi feita aos 120 dias após o transplante.

d) A largura do fruto=LF $(\mathrm{cm})$, obtida através da mensuração da secção transversal do fruto (com um paquímetro) e corresponde ao valor médio da amostragem dos 10 primeiros frutos avaliados por parcela.

e) Comprimento do fruto $=\mathrm{CF}$ $(\mathrm{cm})$, obtido através da mensuração (com uma régua graduada) da secção longitudinal dos mesmos frutos avaliados no ítem " $d$ ".

f) Espessura da polpa=EP ( $\mathrm{mm})$, corresponde à espessura da polpa dos frutos avaliados no ítem " $d$ e $e$ " cortados transversalmente e medidos com uma régua graduada.

g) Número de lóculos=NL, corresponde à contagem do número de - lóculos dos frutos avaliados nos ítens " $d$ e $e$ ".

h) Sólidos solúveis totais=BRIX (\%), determinado por meio do índice refratométrico do suco retirado da cavidade locular dos frutos avaliados nos ítens " $d \mathrm{e} e$ ".

i) Número total de frutos/planta. NTF, obtido por meio da contagem dos frutos produzidos pelas plantas em cada colheita.

j) Peso médio dos frutos/ planta=PMF (g), obtido por meio de pesagem dos frutos (em balança eletrônica) avaliados nos ítens " $d$ e $e$ ".

1) Produção estimada de frutos=PEF (ton/ha), correspondendo ao peso total de 
todos os frutos produzidos pelas plantas de cada parcela.

Estas variáveis foram utilizadas para o desenvolvimento da análise multivariada. A análise de agrupamento envolveu básicamente duas etapas: a estimação de uma medida de similaridade entre os indivíduos e a adoção de uma técnica de agrupamento para se formar os grupos. Neste tarbalho, utilizou-se como medida de similaridade, as Distâncias Generalizadas de Mahalanobis e a técnica de agrupamento baseada no Método de Otimização de Tocher, conforme metodologia preconizada por MAHALANOBIS (1936). Os dados experimentais foram processados em microcomputador utilizando-se os recursos do programa GENES desenvolvido por CRUZ (1990).

\section{RESULTADOS E DISCUSSÃO}

Os grupamentos formados pelo método de Tocher são mostrados na Tabela 1. Por meio deste método de otimização de grupos, a Distância Generalizada de Mahalanobis foi capaz de agrupar as 29 populações de cubiu em nove diferentes grupos: I constituído das populações $4,5,7,9$, $11,13,14,16,17,20,21,24$, e 29 ; II - constituído pelas populações 15 e 19; III - constituído pelas populações 12 , 22 e 27; IV - constituído pelas populações 1,25 e $26 ; \mathrm{V}$ - constituído pelas populações 23 e 28 ; VI constituído pelas populações $2 \mathrm{e} 3$; VII - constituído pelas populações 8 e 10; VIII - constituído exclusivamente pela população 18 e IX - constituído
Tabela 1. Agrupamento das 29 populações de cubiu ( $S$. sessiliflorum) usando as Distâncias Generalizadas de Mahalanobis e o Método de Otimização de Tocher.

\begin{tabular}{lc}
\hline GRUPO & POPULAÇÕES \\
\hline I & $4,5,7,9,11,13,14,16,17,20,21,24$ e 29 \\
II & 15 e 19 \\
III & 12,22 e 27 \\
IV & 1,25 e26 \\
V & 23 e 28 \\
VI & $2 \mathrm{e} 3$ \\
VII & $8 \mathrm{e} 10$ \\
VIII & 18 \\
IX & 6
\end{tabular}

Distância minima $=2,90$ entre as populaçōes 7 e 29 .

Distância máxima $=28,44$ entre as populaçōes 8 e 25 .

exclusivamente pela população 6 .

Pelo número de grupos formados, observou-se que apesar do cubiu estar sendo cultivado em pequena escala nos fundos de quintais de poucos agricultores tradicionais do Amazonas, 9 grupos num universo de 29 populações, pode ser considerado um número bem representativo. RANGEL et al. (1991) observaram em arroz e MALUF (1983) em feijão-vagem, como cultivares dessas espécies estão perdendo a variabilidade ao longo do tempo. Estudos de agrupamento realizado por esses pesquisadores utilizando a Distância Generalizada de Mahalanobis e o Método de Otimização de Tocher, revelaram que num experimento envolvendo 72 cultivares de arroz e 10 cultivares de feijão-vagem, originários de diferentes regiões do mundo, foi possível formar 5 grupos entre cultivares de arroz e 4 cultivares de feijão-vagem. Pelos poucos exemplos mostrados, é muito importante que se promova a manutenção das populações de cubiu existentes no Estado do 
Amazonas, para evitar que a sua variabilidade genética se restrinja a níveis críticos como se tem observado com outras espécies.

A análise das Distâncias Generalizadas de Mahalanobis identificou as populações procedentes de Umariaçú e de São Paulo de Olivença, ambas procedentes do Alto Solimões, como os pares de menor distância genética. Entre os pares de maior distância foram reconhecidas as populações oriundas de Borba e de São Gabriel da Cachoeira, localizadas em bacias hidrográficas totalmente diferentes. Esses resultados são compatíveis com a distribuição geográfica dessas populações na Amazônia (Fig.1).

Ao analisar as divergências intra e inter grupos verifica-se que as posições das populações nos grupos, de maneira geral, ficaram bem representadas. As populações 6 e 20, pela localização geográfica, poderiam formar um mesmo grupo, caso houvesse trocas gênicas entre populações de cubiu; o mesmo poder-se-ia inferir em relação às populações $2 \mathrm{e} 3$, e às peruanas $1,18 \mathrm{e}$ 19. Todavia, elas não apresentaram afinidades pronunciadas, apesar de estarem em localizações geográficas muito próximas (Fig.1). Atribui-se a manutenção dessas divergências genéticas, ao fato do cubiu ser uma espécie autógama e a seleção estar sendo efetuada não sobre caracteres adaptativos, mas, sobre os agronômicos. Na verdade, não é tão simples definir quais as principais características para uma eficiente classificação de genótipos.
Sabe-se que as relacionadas com a adaptação é uma boa escolha. Contudo, MIRANDA et al. (1988) com pimentão e MALUF et al. (1983) com tomate, tiveram bons resultados com análises dialélicas, usando diferenças morfológicas e fisiológicas no estudo de divergências genéticas, empregando a Distância Generalizada de Mahalanobis. A metodologia adotada neste trabalho mostrou-se adequada para avaliar as divergências genéticas entre essas populações de cubiu e os resultados sugerem, sem dúvidas, que em épocas recentes podem ter ocorrido muitos intercâmbios na Amazônia com esse recurso genético deixado pelos ameríndios.

A análise da Figura 2 mostra que, dentro dos 9 grupos formados, aqueles de maiores valores médios de distâncias são os que reuniram um maior número de populações. É plenamente visível que os valores médios das distâncias intragrupos é inferior a qualquer distância intergrupos. Estas constatações estão de acordo com RAO et al. (1985) e CRUZ (1990) quando explicaram que o método de agrupamento de Tocher adota o critério de manter esse comportamento observado nas médias das distâncias calculadas neste trabalho.

Outro aspécto importante, mostrado na Figura 2, são os valores médios das distâncias existentes entre os grupos II e III $(20,01)$, II e IV $(22,74)$, IV e VII $(22,86)$ e entre VIe VII $(22,57)$. As populações que representam esses grupos são aquelas que, em avaliações feitas por SILVA FILHO $e t$ al. (1993), apresentaram as melhores características agronômicas para o 
aproveitamento em melhoramento genético do cubiu. De acordo com WRIGHT (1978), em estudos de divergências genéticas, o melhorista deve selecionar como progenitores para os cruzamentos aquelas populações detentoras das melhores características agronômicas e que apresentarem as maiores diatâncias genéticas entre si. Levando em consideração esses critérios, as populações de Ataláia do Norte (AM), Borba (AM), Iquitos (Peru), São Gabriel da Cachoeira $(A M)$ e a de Belém (PA) foram identificadas como progenitores potenciais para serem utilizados em programa de melhoramento do cubiu, na Zona da Mata do Estado de Pernambuco. FG2

\section{CONCLUSÕES}

A análise da Distância Generalizada de Mahalanobis identificou, entre os pares de menor e maior distâncias genéticas, as populações de cubiu originárias de Umariaçú (AM) e de São Paulo de Olivença (AM) e as de Borba (AM) e de São Gabriel da Cachoeira (AM), respectivamente.

O Método de Otimização de Tocher, utilizando a Distância

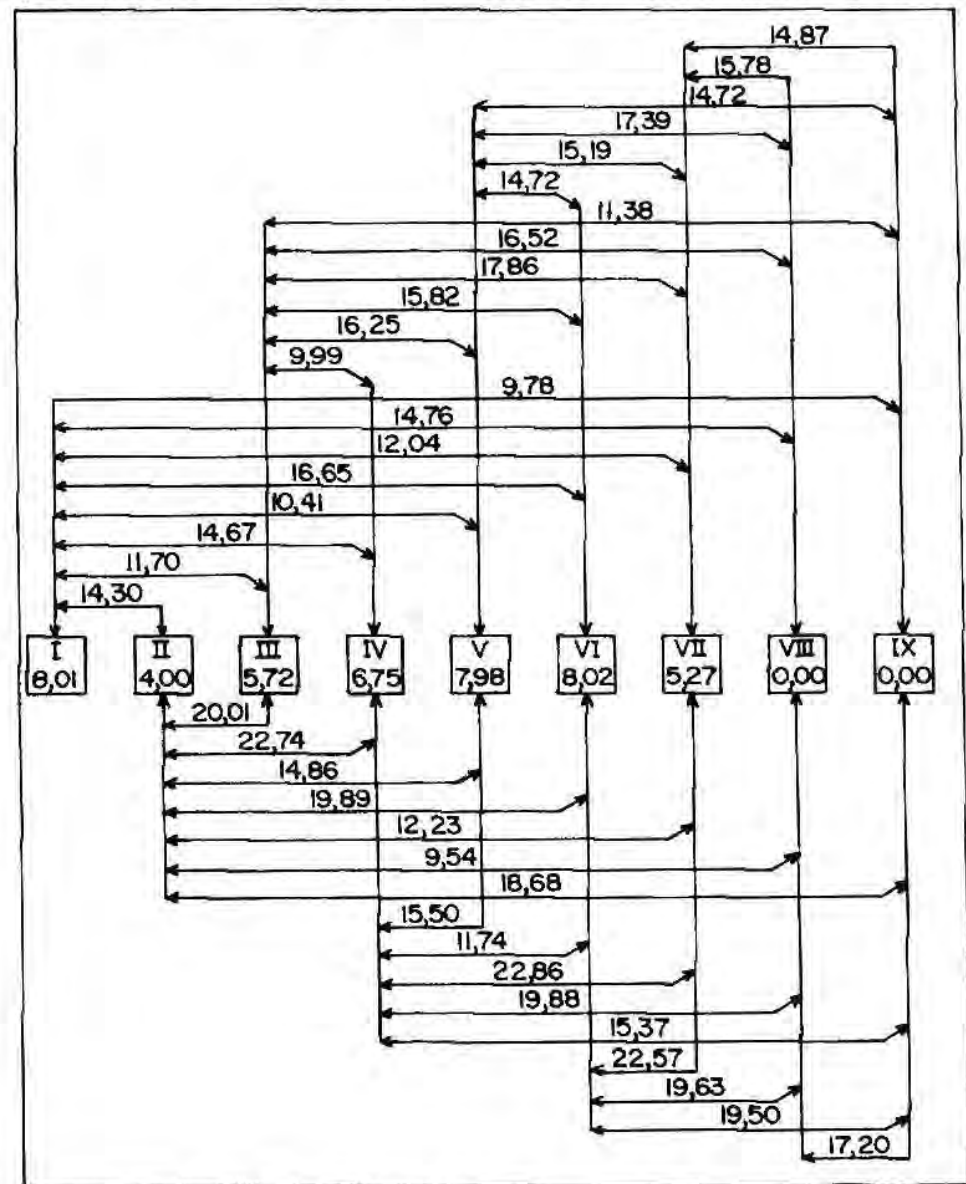

Figura 2. Diagrama resultante da análise inter e intragrupos em 29 populaçōes de cubiu com base na Distância Generalizada de Mahalanobis. 
Generalizada de Mahalanobis, agrupou as 29 populações de cubiu em 9 diferentes grupos.

As populações de cubiu procedentes de Ataláia do Norte (AM), Borba (AM), Iquitos (Peru), São Gabriel da Cachoeira (AM) e de Belém (PA) foram identificadas como as mais divergentes. Portanto, indicadas para o aproveitamento como progenitores em programa de melhoramento genético do cubiu.

O método de agrupamento de otimização de Tocher baseado nas Distâncias Generalizadas de Mahalanobis, é uma técnica eficiente e recomendável para estudar as distâncias genéticas em populações de cubiu.

\section{AGRADECIMENTOS}

Os autores deste trabalho agradecem à Coordenação de Aperfeiçoamento de Pessoal de Nível Superior (CAPES) e à Empresa Pernambucana de Pesquisa Agropecuária (IPA) pela subvenção e o apoio indispensável para que esta pesquisa fosse realizada.

\section{Bibliografia citada}

BEHL, R.K.; SINGH, V.P.; PARODA, R.S. 1985. Genetic divergence in relation to heterosis and specific combining in triticale. Indian Journal Genetic Plant Breeding, 45:368-375.

BAHTT, G.M. 1970. Multivariate analysis approach to selection of parents hibridization aimining at yeld improvement in self-pollinated crops. Australian Journal Agricultural Research, 21:1-7.

CONSELHODE DESENVOLVIMENTO DO ESTADO DE PERNAMBUCO (RECIFE,PE). 1987. Zoneamento pedoclimático do Estado de Pernambuco.
Relatório de dados básicos, Recife: IPA/ SUDENE. v. 1. 183p.

CRUZ, C.D. 1990. Aplicação de algumas técnicas multivariadas no melhoramento de plantas. Piracicaba: ESALQ, 188p. Tese Doutorado.

ENCARNAÇÃO, C.R.F. 1980. Observaçôes meteorológicas e tipos climáticos das unidades e campos experimentais da Empresa Pernambucana de Pesquisa Agropecuária. Recife: IPA, 100 p.

EVANS, G.C. 1982. The quantitative analysis of plant growth. Oxford London: Blackwell Scientific Publications. 387 p.

MAHALANOBIS, P.C. 1936. On the generalized distance in statistic. Procedure National Institute Science, 2:49-55.

MALUF, W.R. 1983. Análise multivariada da divergência genética em feijão-vagem (Phaseolus vulgaris L.). Horticultura Brasileira, 1(2);31-34.

MALUF, W.R,; FERREIRA, P.E;; MIRANDA, J.E.C. 1983. Genetic divergence in tomatoes and its relationship with heterosis for yield in $\mathrm{F}_{1}$ hibryds. Revista Brasileira de Genética, 11:929-937.

MIRANDA, J.E.C.; CRUZ, C.D.; COSTA, C.P. 1988. Predição do comportamento de híbridos de pimentão (Capsicum annuиm L.) pela divergência genética dos progenitores. Revista Brasileira de Genética, 11:929-937.

OLIVEIRA, E.S. 1989. Análise multivariada no estudo da divergência_genética entre cultivares de feijão (Phaseolus vulgaris L.). Viçosa, UVF, 91 p. Tese Mestrado.

PAHLEN, A. von der. 1977. Cubiu (Solanum topiro Humb. \& Bonpl.), uma fruteira da Amazônia. Acta Amazônica, 7:301-307.

RANGEL, P.H.N.; CRUZ, C.D.; VENCOVSKY, R;; FERREIRA, R.P. 1991. Selection of local lowland rice cultivars based on multivariate genetic divergence. Revista Brasileira de Genética, 14(2):437-453.

RAO, C.P.; RAHMAN, M.A.; RAO, P.N.; REDDY, J.R. 1985. Genetic divergence analysis in sugarcane. Genetic Agricultural, 39:237-247. 
SHANSUNDDIN, A.K.M. 1985. Genetic diversity in relation to heterosis and combining ability in spring wheat. Theoret. Appl. Genetic, 70:306-308.

SINGH, T.H.S.S. 1984. Genetic diversity in upland cotton under different environments. Indian Journal Genetic Plant Breeding, 44:506-513.

SILVA FILHO, D.F.; CLEMENT, C.R.; NODA, H. 1989. Variação fenotípica em frutos de doze introduções de cubiu (Solanum sessiliflorum Dunal) avaliadas em Manaus, AM, Brasil. Acta Amazônica, 19:9-18.

SILVA FILHO, D.F.; CLEMENT, C.R.; NODA, H. 1990. Relação entre descritores dos frutos e populações de cubiu (Solanum sessiliflorum Dunal) avaliadas na Amazônia Central. Revista Brasileira de Fruticultura, 10(2):67-70.
SILVA FILHO, D.F.; NODA, H.; CLEMENT, C.R. 1993. Genetic variability of economic characters in 30 accessions of cubiu (Solanum sessiliflorum Dunal) evaluated in Central Amazonia. Revista Brasileira de Genética, 16(2):409-417.

WRIGHT, S. 1987. 1978. Variability within and among natural populations. Chicago: University of Chicago. 2, 580 p. 